\section{Depressed-type colon cancer in a patient with diverticulosis}

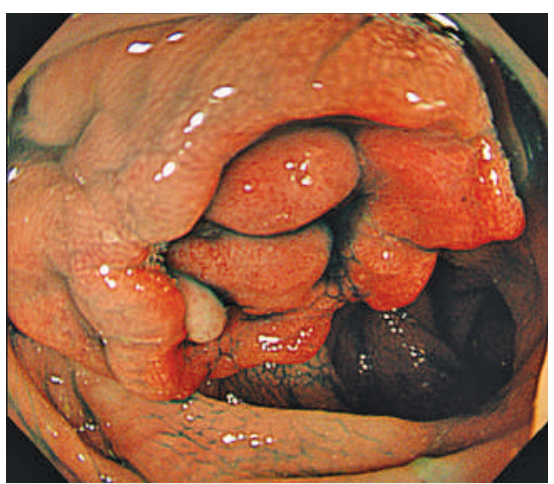

Fig. 1 A depressed lesion surrounded by normal mucosa in the sigmoid colon, visualized by spraying with indigo carmine dye.

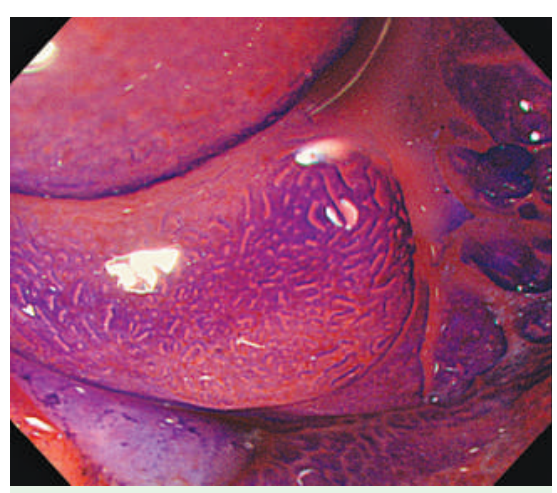

Fig. 2 Magnifying colonoscopy with crystalviolet staining showed type IIIL and type IIIs Kudo pit patterns in the lesion.

A 63-year-old man was referred to our hospital with a history of hematochezia. He had no previous history of abdominal complaints or of abdominal surgery. Physical examination and laboratory parameters showed no abnormalities. Colonoscopy revealed diverticulosis in the sigmoid colon, and a 2-cm-diameter, hard mass within multiple diverticula. The mass was surrounded by normal mucosa, mimicking a submucosal tumor, and an apparently depressed area was identified at the top of the lesion ( $\bullet$ Fig. 1). Because chromoendoscopy using indigo carmine dye clearly showed the margin of the depressed area, the lesion was suspected to be a depressed-type neoplasm, and magnifying colonoscopy with crystal-violet staining showed type IIIL and type IIIs Kudo pit patterns ( $\bullet$ Fig. 2), mainly corresponding to an intramucosal neoplasm; a biopsy sample from the de-
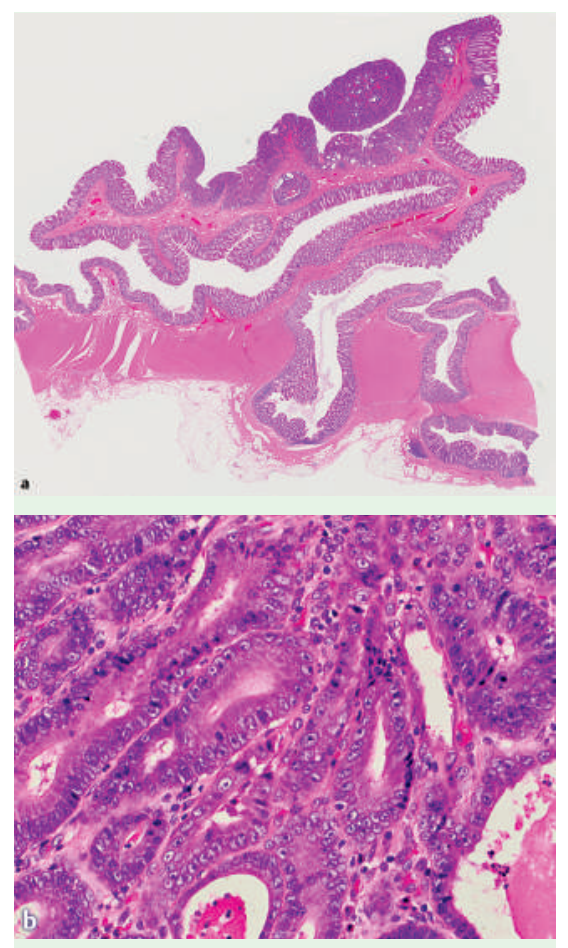

Fig. 3 Histopathological views of the resected specimen after staining with hematoxylin and eosin at original magnification $\times 10$ (a) and at original magnification $\times 200($ b) , showing a depressed-type intramucosal adenocarcinoma with numerous diverticula.

pressed area showed features characteristic of adenoma. The lesion was finally diagnosed as a depressed-type intramucosal neoplasm arising from the diverticulum. Although these lesions are usually treated endoscopically, the coexistence of diverticulosis was thought to pose a high risk of perforation. A sigmoidectomy was therefore performed, and histopathological evaluation of the resected specimen revealed an intramucosal depressed-type adenocarcinoma, $18 \mathrm{~mm} \times 15 \mathrm{~mm}$ in size, accompanied by numerous diverticula (๑ Fig. 3). No evidence of lymph node metastasis was found, and the patient recovered uneventfully.

Although it is still unclear whether diverticular disease is a risk factor for colon cancer, several investigators have described colon cancers associated with diverticulosis or within a diverticulum [1-5]. Because diverticulosis can mask the symptoms of cancer and can also make colonoscopic observation difficult, intramucosal cancer in association with diverticulosis is rarely reported. Moreover, to our knowledge, this is the first report of a depressed-type colon cancer occurring with diverticulosis. Although in this case we were unable to confirm that the cancer had arisen within a diverticulum, we were also unable to explain the unusual appearance of this lesion without reference to the influence of the coexisting diverticulosis.

\section{Endoscopy_UCTN_Code_CCL_1AD_2AB}

\section{N. Kobayashi ${ }^{1}$, K. Hirabayashi ${ }^{2}$,}

T. Matsui ${ }^{3}$, Y. Hirahara ${ }^{1}$, H. Kurihara ${ }^{1}$,

S. Igarashi ${ }^{2}$, T. Ishikawa ${ }^{1,4}$, R. Sekiguchi ${ }^{1}$

1 Department of Diagnostic Imaging, Tochigi Cancer Center, Tochigi, Japan

2 Department of Surgery, Tochigi Cancer Center, Tochigi, Japan

3 Department of Pathology, Tochigi Cancer Center, Tochigi, Japan

${ }^{4}$ Department of Radiology, Dokkyo University School of Medicine, Tochigi, Japan

\section{References}

1 Cohn KH, Weimar JA, Fani K et al. Adenocarcinoma arising within a colonic diverticulum: report of two cases and review of the literature. Surgery 1993; 113: 223-226

2 McCraw RC, Wilson SM, Brown FM et al. Adenocarcinoma arising in a sigmoid diverticulum: report of a case. Dis Colon Rectum 1976; 19: $553-556$

3 Bellows CB, Haque S. Adenocarcinoma within a diverticulum: a common tumor arising in an uncommon location. Dig Dis Sci 2002; 47: 2758-2759

4 Stefánsson T, Ekbom A, Sparèn $P$ et al. Increased risk of left-sided colon cancer in patients with diverticular disease. Gut 1993; 34: 499-502

5 McCallum A, Eastwood MA, Smith AN et al. Colonic diverticulosis in patients with colorectal cancer and in controls. Scand J Gastroenterol 1988; 23: 284-286

Bibliography

DOI 10.1055/s-2007-966854

Endoscopy 2008; 40: E44

(c) Georg Thieme Verlag KG Stuttgart · New York . ISSN 0013-726X

\section{Corresponding author}

\section{N. Kobayashi, MD}

Department of Diagnostic Imaging

Tochigi Cancer Center

4-9-13 Yonan

Utsunomiya

Tochigi 320-0834

Japan

Fax: +81-28-658-5488

nkobayas@tcc.pref.tochigi.jp 